\title{
Rhythm control treatment strategies for atrial fibrillation: current consensus and future possibilities
}

\author{
Author: Ahmed Alsunbuli ${ }^{\mathrm{A}}$
}

\section{Introduction}

Atrial fibrillation (AF) is a disease of ageing, with a prevalence of $3 \%$ among people $>20$ years of age. ${ }^{1,2}$ Around $10-40 \%$ of AF patients are hospitalised annually. Budget-wise, direct AF care consumes $1 \%$ of total health spending in the UK. ${ }^{1} \mathrm{AF}$ is the cause of many debilitating conditions such as stroke and other thrombotic disorders. ${ }^{3}$

\section{Materials and methods}

A systematic search for evidence was carried out by investigating online resources: MEDLINE, CINAHL, PUBMED, EMBASE, ScienceDirect and HDAS. Terms used in research included 'rhythm control', 'management strategies', 'AAD', 'antiarrhythmic strategies', 'atrial fibrillation', 'AF', 'ablat*', 'non-pharmacological'. A specific search was done through National Institute for Health and Care Excellence (NICE), European Society of Cardiology (ESC), American College of Cardiology (ACC) / American Heart Association (AHA) guidelines as well. Research was limited to the past 10 years to provide more contemporary evidence.

\section{Results and discussion}

For infrequent paroxysms, a 'no-drug-treatment' strategy or a 'pill-in-the-pocket' strategy is followed. ${ }^{4}$ In heart failure patients, both amiodarone and sotalol are favoured, whereas dronaderone is contraindicated. Also, in ischaemic or structural heart disease, the use of class $1 \mathrm{C}$ antiarrhythmic drugs (AADs) is not recommended (Fig 1). ${ }^{3}$ Komatsu et al noted that disopyramide is more effective for night-time AF while flecainide and pilsicanide are better for daytime episodes. The main challenge with AADs is their adverse effects profile. ${ }^{5}$

Catheter ablation (CA) techniques, when compared with AADs, have better quality of life outcomes and fewer clinical events and are usually recommended after a failed trial of AAD treatment. ${ }^{3,6,7}$ There are different techniques for CA but pulmonary vein isolation remains the cornerstone of treatment. ${ }^{8}$ The main limitation for CA treatment is its anatomically sinister complications and adverse events.

Surgical ablation (SA), whether open or thoracoscopic, showed better freedom-from-AF profile when compared

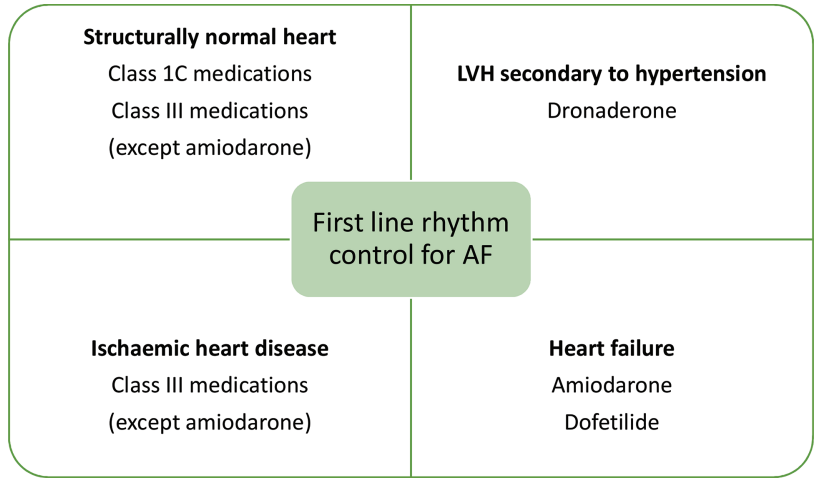

Fig 1. Rhythm control strategies in atrial fibrillation; second line treatment is catheter ablation and then, if unsuccessful, amiodarone can be started. ${ }^{10}$ Class III medications = dronedarone, dofetilide and sotalol; $\mathrm{LVH}=$ left ventricular hypertrophy.

with CA but with almost double the rate of adverse events. ${ }^{9}$ It can have a role in concomitant mitral valve surgery and AF maze operation. ${ }^{9}$ The main challenge with SA is the lack of well-conducted randomised trials, and also in the differences between rhythm monitoring protocols which may result in different measured outcomes. Hybrid treatment approaches show promise in tackling AF rhythm therapy.

Generally, the consensus of NICE, ESC, ACC/AHA recommends that an initial trial of AADs is followed, if failed or contraindicated, by CA. A surgical approach should be considered if there is a concomitant cardiac surgery or after two failed CA attempts. ${ }^{3,6,7}$

\section{Conclusion}

There is no clear best method for rhythm control treatment, which is further complicated by a significant gap in evidence for different ablation and surgical techniques. Although hybrid approaches can give hope for better outcomes, there needs to be more research to determine that potential. Finally, a remarkable opportunity exists for researchers in AF as illustrated in this study, which can be tackled in future research for better clinical outcomes in AF patients.

\section{Conflicts of interest}

None declared. 


\section{References}

1 Chugh SS, Havmoeller R, Narayanan K et al. Worldwide epidemiology of atrial fibrillation: a Global Burden of Disease 2010 Study. Circulation 2014;25;129:837-47.

2 Bjorck S, Palaszewski B, Friberg L, Bergfeldt L. Atrial fibrillation, stroke risk, and warfarin therapy revisited: a population-based study. Stroke 2013;44:3103-8.

3 Kirchhof P, Benussi S, Kotecha D et al. 2016 ESC guidelines for the management of atrial fibrillation developed in collaboration with EACTS. Eur Heart J 2016;37:2893-962.

4 Alboni P, Botto GL, Baldi N et al. Outpatient treatment of recentonset atrial fibrillation with the 'Pill-in-the-pocket' approach. $N$ Engl J Med 2004;351:2384-91.

5 Komatsu T. Current strategies of antiarrhythmic drug therapy for paroxysmal atrial fibrillation. J Arrhythm 2012;28:162-9.
6 National Institute for Health and Care Excellence. Atrial fibrillation: Management. London: NICE; 2014.

7 January CT, Wann LS, Alpert JS et al. 2014 AHA/ACC/ HRS guideline for the management of patients with atrial fibrillation: A report of the American College of Cardiology/ American Heart Association Task Force on practice guidelines and the Heart Rhythm Society. J Am Coll Cardiol 2014;64:e1-76.

8 Kalla M, Sanders P, Kalman JM, Lee G. Radiofrequency catheter ablation for atrial fibrillation: Approaches and outcomes. Heart Lung Circ 2017;26:941-9.

9 Huffman MD, Karmali KN, Berendsen MA et al. Concomitant atrial fibrillation surgery for people undergoing cardiac surgery. Cochrane Database Syst Rev 2016:CD011814.

10 Piccini JP, Fauchier L. Rhythm control in atrial fibrillation. Lancet 2016;388:829-40. 